\title{
Involvement of gicerin, a cell adhesion molecule, in a dermal autograft chicken model
}

\author{
YASUHIRO TSUKAMOTO ${ }^{1}$, BIN KATAOKA ${ }^{2}$, KAZUHIDE ADACHI ${ }^{1}$, HIDENORI KATO ${ }^{1}$, \\ MARIE INAI $^{1}$, MASAYA TSUKAMOTO ${ }^{1}$, EKOWATI HANDHARYANI ${ }^{3}$ and EIICHI TAIRA ${ }^{4}$ \\ ${ }^{1}$ Department of Animal Hygiene, Graduate School of Environmental and Biological Sciences, Kyoto Prefecture University, \\ Kyoto; ${ }^{2}$ Department of Veterinary Anatomy, Graduate School of Environmental and Biological Sciences, \\ Osaka Prefecture University, Osaka, Japan; ${ }^{3}$ Faculty of Veterinary Medicine, Bogor Agriculture University, \\ JL. Agatis, Kampus IPB Darmaga, Bogor, Indonesia; ${ }^{4}$ Department of Pharmacology, \\ Graduate School of Medicine, Iwate Medical University, Iwate, Japan
}

Received May 31, 2010; Accepted June 29, 2010

DOI: 10.3892/ijmm_00000511

\begin{abstract}
Gicerin is a cell adhesion molecule in the immunoglobulin superfamily. This molecule has homophilic and heterophilic adhesive activities, binding to the neurite outgrowth factor (NOF). We have previously reported that gicerin plays an important role in the development and regeneration as well as in the metastasis of tumors through its adhesive activities, mediating cell-cell and/or cell-extracellular matrix interactions. In this study, we investigated the involvement of gicerin in a dermal autograft chicken model. Gicerin and NOF were transiently present in the regenerating epithelia after the dermal graft transplantation. The treatment with an anti-gicerin polyclonal antibody, by placing drops onto the wounds, inhibited the adhesiveness of the grafts to the marginal skin. The chimeric protein of gicerin-IgG, gicerin-Fc, and NOF proteins promoted the regeneration of the grafts. These findings suggest the potential function of gicerin in dermal autografts, and gicerin and NOF proteins could help clinical improvement after transplantations.
\end{abstract}

\section{Introduction}

Adhesion among cells is an essential property of multicellular organisms for constructing tissues and organs, as well as for establishing and maintaining cell-cell interactions $(1,2)$. Cell adhesion molecules (CAMs) have been recognized to play a major role in a variety of physiological and pathological phenomena, including the regulation of organogenesis,

Correspondence to: Professor Yasuhiro Tsukamoto, Department of Animal Hygiene, Graduate School of Biology and Environmental Sciences, Kyoto Prefecture University, 1-5 Nakaragicho, Kyoto 606-8522, Japan

E-mail: ytsuka@kpu.ac.jp

Key words: gicerin, cell adhesion molecule, neurite outgrowth factor, chicken, autograft, regeneration, skin maintenance of tissue architecture, wound healing, as well as tumor invasion and metastasis (3-5).

The neurite outgrowth factor (NOF) is an extracellular matrix (ECM) glycoprotein purified from chicken gizzard smooth muscle and promotes neurite extension from neurons $(6,7)$. Gicerin is an integral membrane protein in the immunoglobulin (Ig)-CAM superfamily, purified as a ligand of NOF from the chicken gizzard muscle. The deduced amino acid sequence of gicerin indicates that this molecule has 5 Ig-like loops in its extracellular domain and small cytoplasmic tail (8). We have previously reported that gicerin plays an important role in the development and regeneration of the nervous system and epithelial tissue through its adhesive activities, mediating cell-cell and/or cell-ECM interactions (9-13).

As the histology of chicken skin is quite simple and much thinner (it consists of the monostratal basal layer, 1-2 layers, the stratum spinosum epidermidis, and the very thin corneum and dermis) than that of mammals, it has therefore been utilized as an excellent model for both dermal regeneration and thick-split grafting $(14,15)$. In a preliminary experiment, we found an increased expression of gicerin and its ligand during dermal regeneration. Gicerin was present on the cell surface of the epithelial cells, and NOF was localized in the basement membrane. Therefore, we consider that the adhesive activities of gicerin could play an important role in dermal regeneration.

In this study, we aimed to i) set up a chicken dermal autograft model and examine the appearance of gicerin and NOF in the remodeling process in the grafts, ii) confirm the potential function of gicerin in autografting by applying the anti-gicerin and anti-NOF antibodies onto the wounds, and iii) employ these 2 molecules to improve the progression of the grafts.

\section{Materials and methods}

The dermal autograft chicken model. In the present study, we used white leghorn chickens at 3 weeks of age as the experimental animals. While the chickens were under deep anesthesia, we punch-crafted 2 spots, about $6 \mathrm{~mm}$ in diameter, on the dorsal skin of each one. Next, the autograft was placed 


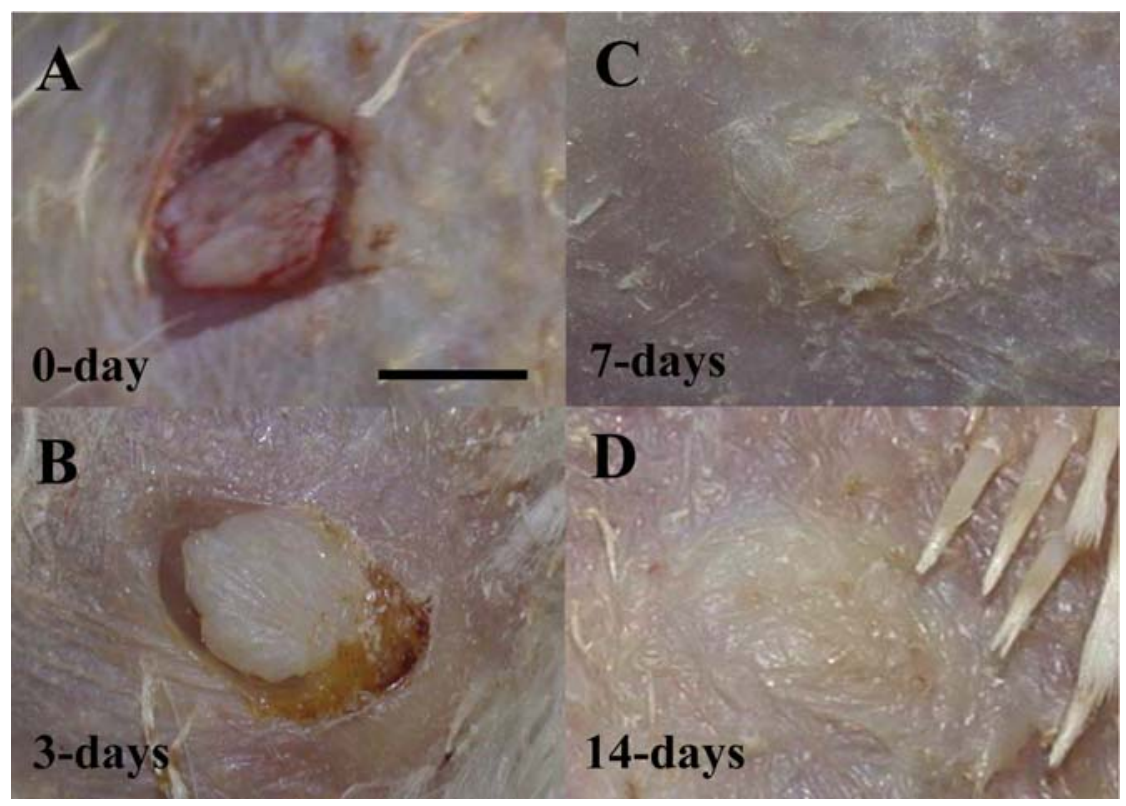

Figure 1. The autografts of the chickens after transplantation. Photographs show the gross appearance of the chicken skin autografts at the time just after implantation, and at 3,7 and 14 days. Bars, $6 \mathrm{~mm}$.

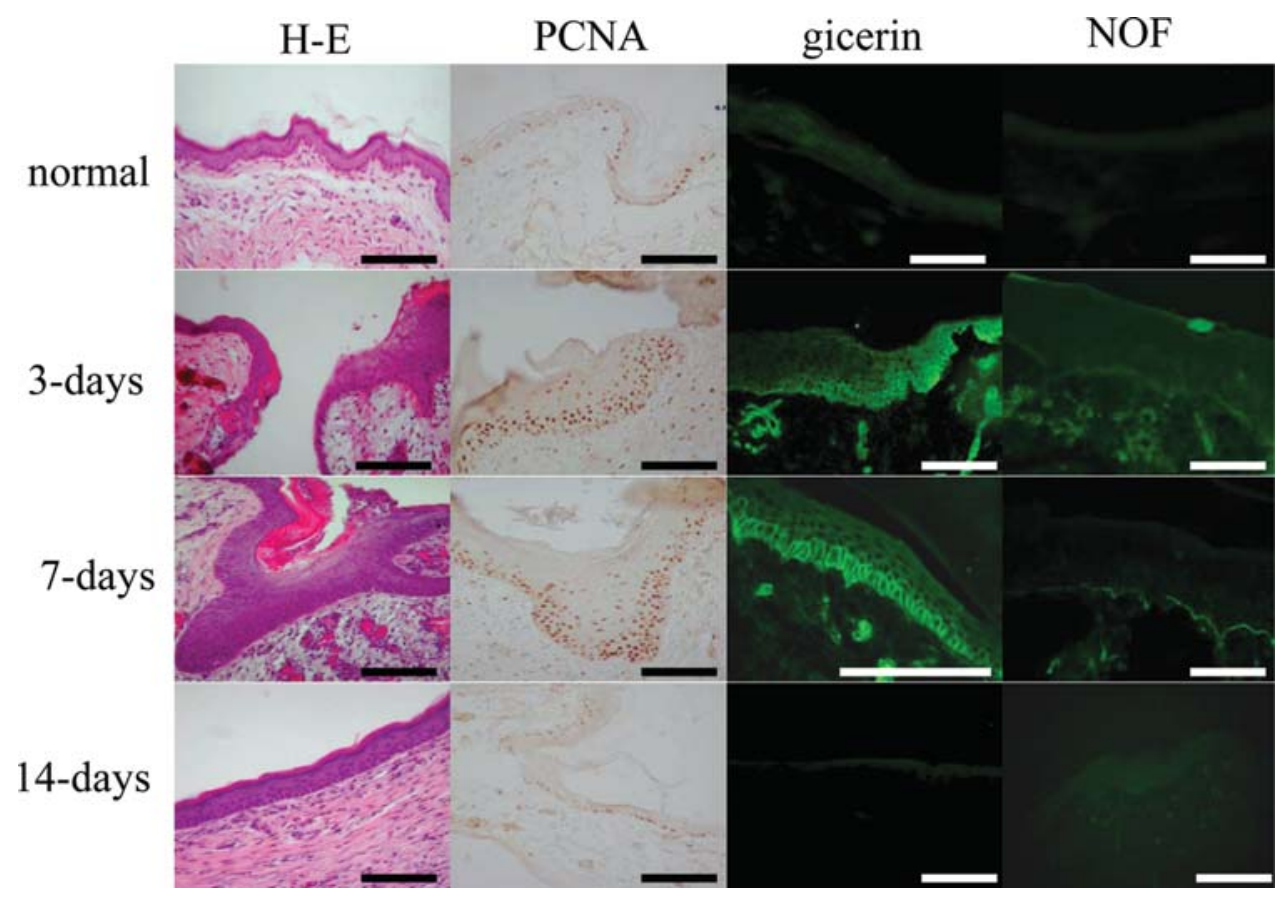

Figure 2. Histopathology of the autografts after transplantation. The sections of normal chicken skin and the autografts at 3, 7, and 14 days p.t., were cut and stained with H\&E. In addition, immunohistochemical staining for PCNA, gicerin and NOF was also performed on the sections. Bars, $50 \mu \mathrm{m}$.

on another wound. At 3, 7 and 14 days post-transplan-tation (p.t.), the birds were sacrificed under deep anesthesia and the grafts with margins were removed immediately. The experiments were approved by the Animal Care Committee of Osaka Prefecture University.

Histopathology. All the dermal samples were fixed with Zamboni's fixative solution. For histopathology, the paraffin sections were cut and stained with hematoxylin and eosin $(\mathrm{H} \& \mathrm{E})$ as a routine procedure. In order to determine the cell proliferation activity, PCNA staining was performed as follows: Following incubation with $3 \% \mathrm{H}_{2} \mathrm{O}_{2}$ in methanol, the post-deparaffinized sections were boiled in a microwave and blocked with $2 \%$ skim milk in phosphate buffered saline (PBS). Thereafter, the samples were incubated with an antiPCNA monoclonal antibody, PC10 (Santa Cruz Biotechnology), for $1 \mathrm{~h}$ at $37^{\circ} \mathrm{C}$ and reacted with a horseradishperoxidase (HRP)-conjugated anti-mouse $\operatorname{IgG}$ for $1 \mathrm{~h}$ at $37^{\circ} \mathrm{C}$. Finally, the immunoreactivity was visualized with $\mathrm{DAB}$ solution (Dako, Japan). 


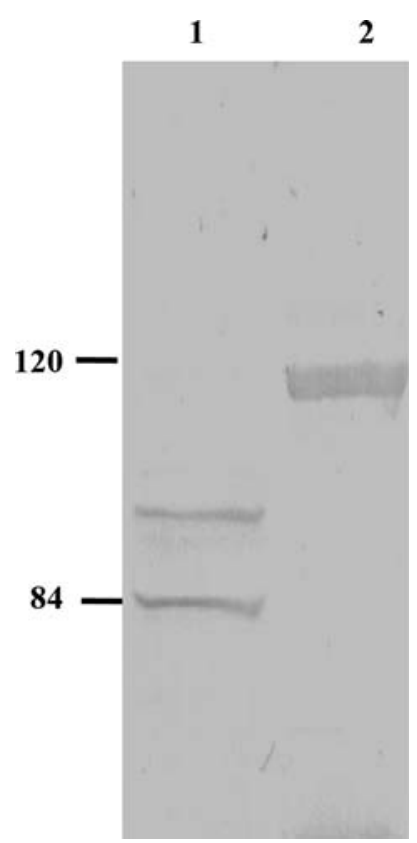

Figure 3. Western blot analysis for the gicerin-Fc chimeric protein. Lane 1 , native gicerin from the gizzard of chickens as the positive control; lane 2, the culture medium from culture dishes showing COS7 growth. Each lane is loaded with $20 \mu 1$ medium under a reducing condition. Note that gicerin-Fc is slightly heavier than native gicerin.

Immunohistochemistry for gicerin and NOF. After washing in an equivalent of $30 \%$ sucrose solution, the Zamboni'sfixed samples were frozen and then cut into $10-\mu \mathrm{m}$ sections using a cryostat. After washing the tissue samples twice with PBS, the sections were incubated with a polyclonal rabbit antibody for gicerin or NOF for $1 \mathrm{~h}$ at $37^{\circ} \mathrm{C}$ (12). After washing twice with PBS, the proteins were incubated with a fluorescein isothiocyanate-conjugated goat anti-rabbit Ig (Dako) for $1 \mathrm{~h}$ at $37^{\circ} \mathrm{C}$. After washing with PBS, they were examined under a fluorescent microscope.

Effects of anti-gicerin and anti-NOF antibodies on the autografts. At 3 days p.t. onto the wounds, $100 \mu \mathrm{l}$ of antigicerin polyclonal antibody $(4 \mathrm{mg} / \mathrm{ml})$ or anti-NOF polyclonal antibody $(4 \mathrm{mg} / \mathrm{ml})$ were dropped onto the grafts. After 4 days (7 days post-autografting), the birds were sacrificed under deep anesthesia, and the autografts with their margins were removed immediately and fixed in Bouin's solution for histopathological examination.

Purification of gicerin-Fc chimeric protein. We previously constructed a plasmid encoding the extracellular domain of gicerin and the human $\mathrm{IgG}-\mathrm{Fc}$ fraction following an elongation factor on the promoter region of the cytomegalovirus (16). This construct was transfected into the monkey kidney cell line, COS7, using the Fugene 6 Transfection Reagent (Roche). After 2 days of culture in GIT-medium, the resulting gicerinFc protein in the culture medium was confirmed by Western blot analysis using the anti-gicerin antibody (see blow). Next, $\sim 400 \mathrm{ml}$ of medium were incubated with $10 \mathrm{mg}$ of protein A-sepharose (Pharmacia) at $4^{\circ} \mathrm{C}$ with gentle rotating. The protein A was then trapped on disposal columns, and the culture medium was allowed to run off. Finally, the gicerin-Fc proteins binding onto protein A were eluted with glycine$\mathrm{NaOH}$ buffer (pH 9.0) after washing with PBS (pH 7.4), and neutralized in $1 \mathrm{M}$ Tris- $\mathrm{HCl}(\mathrm{pH} \mathrm{3.0)}$ buffer. The protein concentration was measured at $568 \mathrm{nM}$ using a BCA kit (Pharmacia). The fraction with the highest concentration of gicerin-Fc was applied to the autograft.

Western blot analysis. The COS7-transfectants with the gicerin-Fc plasmid were separated by $7.5 \%$ sodium dodecyl sulfate-polyacrylamide gel electrophoresis (SDS-PAGE) under reducing conditions, and were electrotransferred onto Bio-Rad polyvinyl difluoride membranes. The blots were blocked with $2 \%$ skim milk in PBS containing $0.05 \%$ Tween-20 (PBST) for $1 \mathrm{~h}$ at $37^{\circ} \mathrm{C}$ and then incubated with a rabbit polyclonal antibody against gicerin diluted in PBST with $2 \%$ skim milk for $1 \mathrm{~h}$ at $37^{\circ} \mathrm{C}$. After being washed with PBST 3 times, and incubated with the HRP-conjugated secondary antibody to rabbit IgG (Dako) in PBST with $2 \%$ skim milk for $1 \mathrm{~h}$ at $37^{\circ} \mathrm{C}$, the samples were washed again 3 times in PBST and then further washed in PBS, and the blots were visualized on X-ray film using an ECL solution (Gibco BRL) (9).

Effects of gicerin-Fc and NOF proteins on the autografts. Gicerin-Fc $(40 \mu \mathrm{g})$ or NOF protein $(50 \mu \mathrm{g})$ were incubated with $0.05 \%$ poly-D-lysine (Invitrogen) for $30 \mathrm{~min}$ at room temperature. These inoculums were applied onto the autografts at 3 days p.t. After another 4 days, the birds were sacrificed under deep anesthesia, and the grafts with marginal tissues were removed and fixed in Bouin's solution for histopathological examination.

\section{Results}

Histopathology of the autografts. Fig. 1 shows the gross appearance of the autografts on the wounds. At the time of transplantation, the grafts were put onto the wounds with considerable non-heading margins (Fig. 1A). At 3 days p.t., the grafts were somewhat adhesive, but the gap was still visible in the margins (Fig. 1B). At 7 days p.t., the grafts had tightly adhered to the margins and the gap was no longer obvious, and the marginal edges were thicker than the normal site (Fig. 1C). At 14 days p.t., the skin with the autografts and their margins had recovered to its normal appearance, although the feathers did not fully re-grow (Fig. 1D).

The histopathology and immunohistochemistry of the autografts are shown in Fig. 2. Histologically, normal chicken skin is constructed with the basal lamina on the basement membrane and a thin corneum on the horny layer and a very thin dermis. PCNA staining was found in the nuclei of a small number of basal cells in normal epithelia. At 3 days p.t., the gap widened between the grafts and marginal tissues, but the epithelial cells at this site were hyperplastic, and numerous PCNA-positive nuclei were observed in the epithelia. This indicated the presence of rapid cell proliferation in the regenerating epithelia. At 7 days p.t., the autografts had adhered completely to the marginal epithelia. The adhesive epithelial edge was hyperplastic and possessed many PCNA-positive nuclei, especially in the basal cells. At 14 days p.t., the stratification of the epithelia ceased, and the 


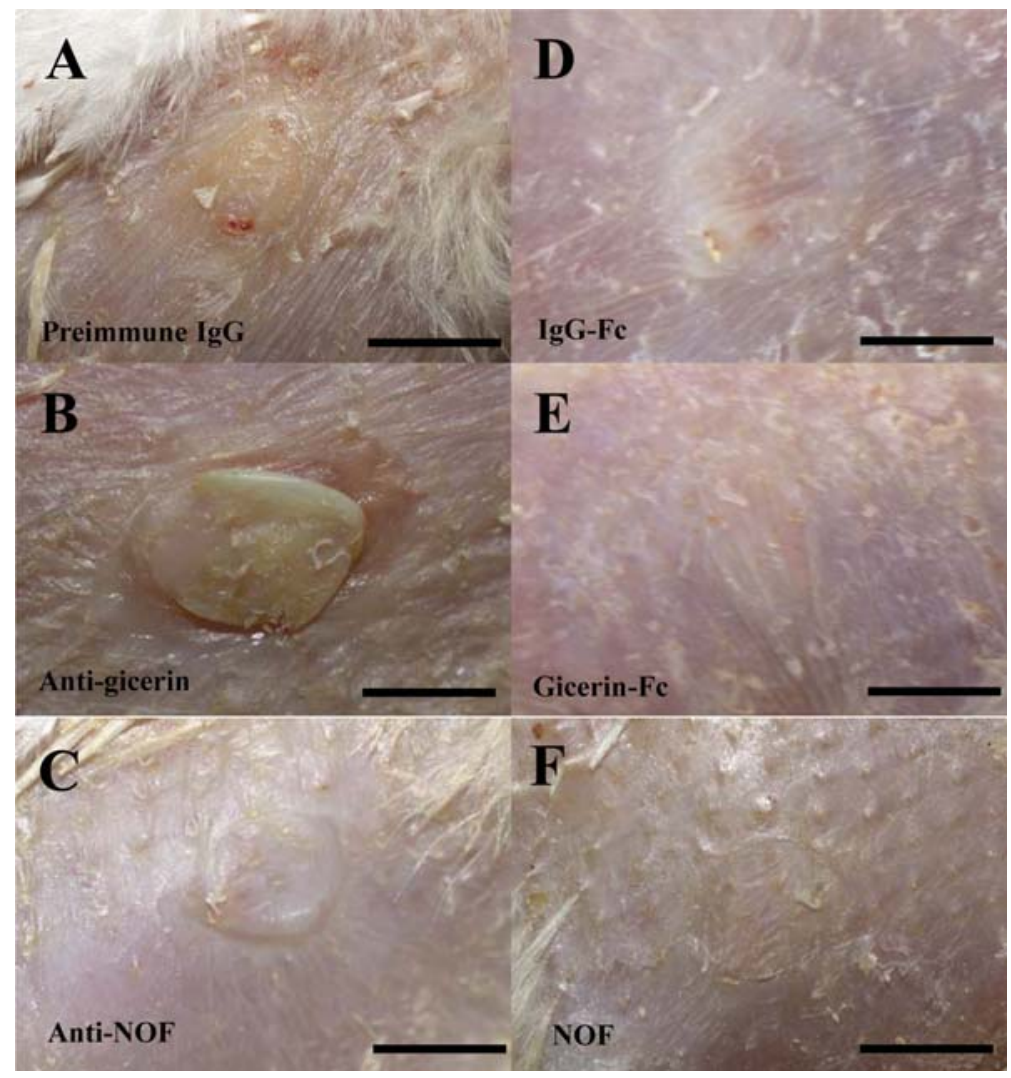

Figure 4. The autografts after treatment with antibodies or proteins. Photographs show the gross appearance of the autografts treated with pre-immune rabbit IgG (pre-immune IgG) (A), with anti-gicerin polyclonal antibody (anti-gicerin) (B), with anti-NOF polyclonal antibody (anti-NOF) (C), with human IgG-Fc $(\mathrm{IgG}-\mathrm{Fc})$ as the control (D), with gicerin-Fc chimeric protein (gicerin-Fc) (E), and with NOF protein (F). Bars, $6 \mathrm{~mm}$.
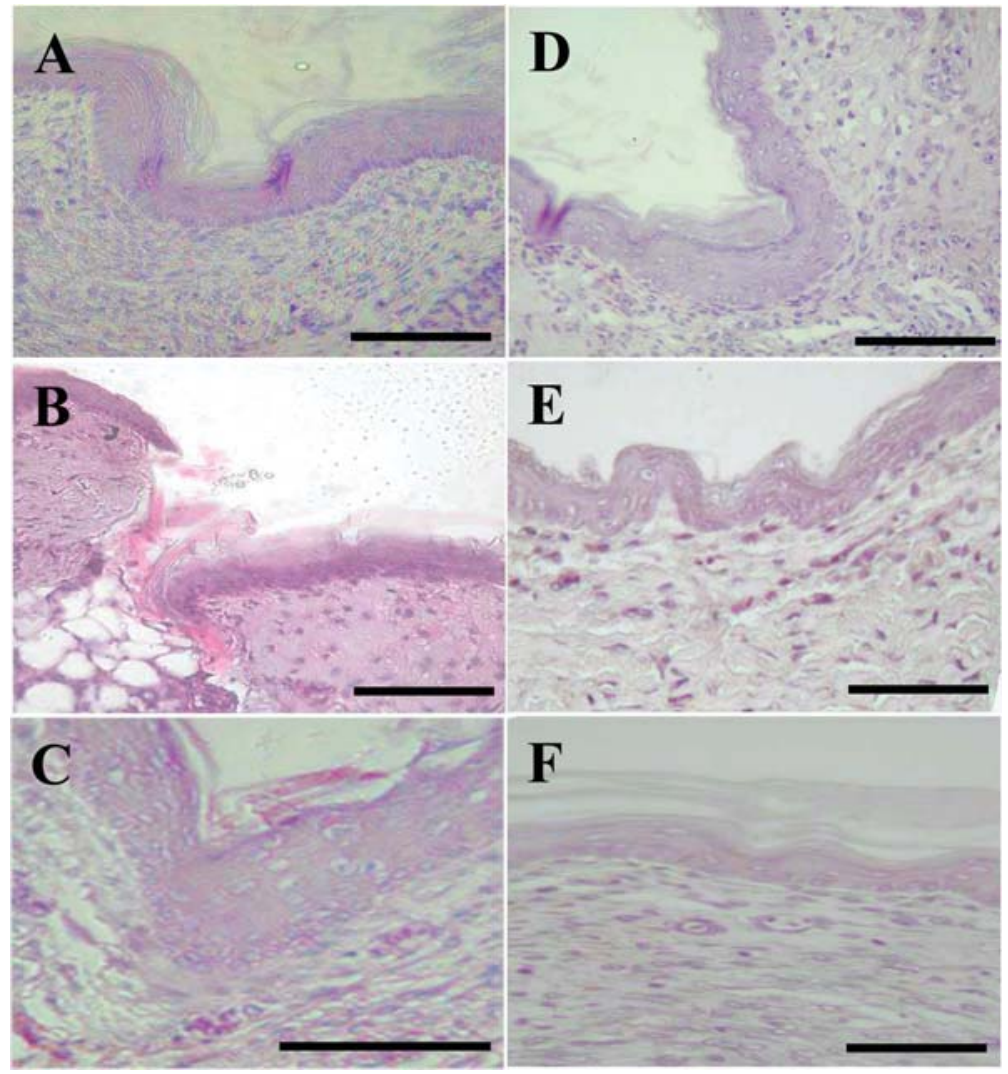

Figure 5. Histopathology of the autografts after treatment with antibodies or proteins at 3 days p.t. The sections of the autografts treated with pre-immune rabbit IgG (A), with anti-gicerin polyclonal antibody (B), with anti-NOF polyclonal antibody (C), with human IgG-Fc as the control (D), or with gicerin-Fc chimeric protein (E), and with NOF protein (F), were cut and stained with H\&E. Bars, $50 \mu \mathrm{m}$. 
epithelium returned to almost normal. The adhesive sites of the epithelia were not distinguishable at this point. The number of PCNA-positive nuclei had also returned to normal. Thus, the epithelial regeneration was almost complete by day 14 .

Expression of gicerin and NOF in the autografts after transplantation. Gicerin and NOF were visualized immunohistochemically on the frozen sections from the autografts at various time-points (Fig. 2). Both gicerin and NOF were barely detectable on the normal epithelia of the chicken skins. However, at 3 and 7 days p.t., the expression level of these 2 molecules was dramatically increased in the epithelia. Gicerin was present in the hyperplastic epithelium, especially in the basal cells, while NOF was present in the basement membrane. Thereafter, at 14 days p.t., the expression of gicerin and NOF was decreased in the grafts and was no longer visible. Therefore, gicerin and NOF were only transiently present in the regenerating epithelia of the autografts.

Effects of anti-gicerin and anti-NOF antibodies on the epithelial regeneration of the autografts. Anti-gicerin and antiNOF antibodies were dropped onto the autografts at 3 days p.t, and the histological sections were prepared after another 4 days. Grossly, the grafts treated with pre-immune IgG showed a normal regenerating process, and the epithelial gap had disappeared (Fig. 4A). However, treatment with the antigicerin antibody inhibited the regeneration, and the gap was clearly visible (Fig. 4B). In contrast, the autografts treated with the anti-NOF antibody underwent normal regeneration (Fig. 4C).

Histopathologically, the epithelia of the autografts treated with the pre-immune IgG completely adhered to the marginal skin, the adhesive epithelial edge was hyperplastic, and was similar to the epithelia of the non-treated grafts (Fig. 5A). Therefore, the pre-immune IgG did not affect the autografting. In contrast, treatment with the anti-gicerin antibody perturbed the autografting at the histological level. The epithelial edges of the grafts did not fuse with the marginal epithelia, and there were outstanding non-heading legions (Fig. 5B). Slight exudation was also found at this site. The anti-NOF antibody did not lead to any apparent abnormality in the graft regeneration (Fig. 5C). We examined the skin from at least 5 birds for each test, and found little variation among the animals.

Effects of gicerin-Fc and NOF proteins on the epithelial regeneration of the autografts. We first had to produce a gicerin chimeric protein, as functional native gicerin from chicken gizzards cannot be obtained due to the rigorous solubilization with NP-40 and SDS that is required. In the present study, gicerin-Fc protein was successfully produced by COS7 cells and purified with protein A. In Western blot analysis, this chimeric protein was recognized as a single band of $120 \mathrm{kDa}$, which was slightly heavier than the native gicerin in the gizzard, because of the additional human Fc fraction (Fig. 3).

The gicerin-Fc and NOF proteins were dropped onto the 3-day-p.t. autografts, and the histological sections were prepared 4 days later. The Fc fraction of human IgG as the control protein did not affect the regeneration process of the autografts by either macroscopic or histological examination (Figs. 4D and 5D). At both the gross and histological levels, the gicerin-Fc and NOF proteins improved regeneration. The grafts fused completely with the marginal skin, the boundaries were not visible, and hyperplasia had already ceased and the epithelial layer had returned to normal (Figs. 4E and F; $5 \mathrm{E}$ and $\mathrm{F}$ ). The epithelial layer with gicerin-Fc and NOF was clearly thinner and almost flattened. This tendency was most remarkable in the grafts with the NOF protein.

\section{Discussion}

In our previous study, we demonstrated that gicerin promotes cell migration and epithelialization $(12,17)$. Epithelial regeneration requires several critical processes: i) Cell migration from the basement membrane, followed by coverage on the non-heading site, ii) epithelial hyperplasia following active cell proliferation, and iii) epithelial structural remodeling. Several molecules, such as growth factors, cytokines, ECM proteins and CAMs, are known to be involved in dermal regeneration (18). Cell-cell and cell-basement membrane interactions by ECM and CAM are especially important phenomena of normal wound healing.

In the present study, we first established an autograft chicken model. In this model, gicerin was present in the regenerating epithelial cells (especially basal cells) of the grafts just adjacent to the marginal epithelium, and NOF was expressed in the epithelial basement membrane at same stage. It is known that gicerin and NOF are produced by epithelial cells and localize in the cell and basement membrane, respectively (17). Accordingly, we speculate that the epithelial cells at the regenerating edges could produce NOF and migrate onto the naked basement membrane as a result of the gicerin-NOF interaction, thus resulting in the coverage of the epithelial gaps. As a newly discovered step, cell-cell interactions by gicerin-gicerin binding could participate in the epithelial adhesion of autografts with marginal skin, also in subsequent epithelial hyperplasia.

In order to confirm these hypotheses, we initiated the treatment of the gaps surrounding the autografts with an antigicerin polyclonal antibody, and found that the epithelial regeneration (fusion of autografts with marginal skin) was highly disturbed. This indicates that gicerin is critical to the autografting process. In contrast, the anti-NOF antibody did not inhibit epithelial regeneration. Similar results were reported in a sciatic nerve regeneration model in which the anti-NOF antibody did not have any effect on the neurite outgrowth from dorsal root ganglions exposed to the NOF protein (16). We believe that since NOF is a large molecular protein that forms trimers (17), the antibody cannot mask all of the functional sites, so the epitope for gicerin remains available. However, further studies, including the use of another inhibition assay, are required to elucidate the possible function of NOF in dermal regeneration.

One particularly interesting and potentially important finding from the present study was the observation that gicerin-Fc and NOF proteins can promote autografting. Previously, we confirmed the functional activity of these 2 proteins by in vitro bioassays or by ligand blotting. Gicerin-Fc can promote cell migration and neurite extension, while the 
NOF protein can bind to the gicerin protein directly and promote neurite projection and cell migration $(9,11,16,19)$. However, this is the first study confirming the potential function of these proteins in an in vivo system. While fibronectin and other ECM proteins are now used for wound healing at clinical levels (18), there have not been any reports concerning the functions of CAMs on injuries.

The present study focused only on epithelial regeneration. However, the epithelial dermal interactions are also critical to the autografting process. In the future, we aim to perform additional studies on the role of gicerin in dermal remodeling. In conclusion, the findings from this study are promising, and suggest that gicerin could have a potential function in the healing of dermal autografts. In addition, our results suggest that the gicerin and NOF proteins could improve clinical outcome after transplantation. Further studies examining the importance of the effects of dose and time on the promotion of autograft adhesion, are therefore considered to be necessary.

\section{Acknowledgements}

This study was supported in part by a Grant-in-Aid for Scientific Research from the Ministry of Education, Science, Sports and Culture, Japan (no. 21380182).

\section{References}

1. Edelman GM: Cell adhesion and the molecular processes of morphogenesis. Annu Rev Biochem 54: 135-169, 1985.

2. Rutishauser U, Acheson A, Hall AK, Mann DM and Sunshine J: The neural cell adhesion molecule (N-CAM) as a regulator of cell-cell interactions. Science 240: 53-57, 1988.

3. Koukoulis GK, Patriarca C and Gould VE: Adhesion molecule and tumor metastasis. Hum Pathol 29: 889-892, 1998.

4. Gallin WJ, Choung CM, Finkel LH and Edelman GM: Antibodies to liver cell adhesion molecule perturb inductive interactions and alter feather pattern and structure. Dev Biol 83: 8235-8239, 1986.

5. Choung CM and Cheng HM: Enhanced expression of neural cell adhesion molecules and tenascin (cytotactin) during wound healing. Am J Pathol 138: 427-440, 1991.

6. Hayashi Y and Miki N: Purification and characterization of a neurite outgrowth factor from chicken gizzard smooth muscle. J Biol Chem 260: 14269-14278, 1985.
7. Taira E, Takaha $\mathrm{N}$ and Miki N: Extracellular matrix proteins with neurite promoting activity and their receptors. Neurosci Res 17: 1-8, 1993 .

8. Taira E, Takaha N, Taniura H, Kim CH and Miki N: Molecular cloning and functional expression of gicerin, a novel cell adhesion molecule that binds to neurite outgrowth factor. Neuron 12: 861-872, 1994.

9. Tsukamoto Y, Taira E, Kotani T, Yamate J, Wada S, Takaha N, Miki N and Sakuma S: Involvement of gicerin, a cell adhesion molecule, in tracheal development and regeneration. Cell Growth Differ 7: 1761-1767, 1996.

10. Tsukamoto Y, Taira E, Yamate J, Nakane Y, Kajimura K, Tsudzuki M, Kiso Y, Kotani T, Miki N and Sakuma S: Gicerin, a cell adhesion molecule, participates in the histogenesis of retina. J Neurobiol 33: 769-780, 1997.

11. Tsukamoto Y, Matsumoto T, Taira E, Kotani T, Yamate J, Takaha N, Tatesaki R, Namikawa T, Miki N and Sakuma S: Adhesive ability of gicerin, a cell adhesion molecule, in kidneys and nephroblastomas of chickens. Cell Tissue Res 292: 137-142, 1998.

12. Tsukamoto Y, Taira E, Kajimura K, Yamate J, Kotani T, Amin H, Kohama K, Sakuma S, Miki N and Sasaki F: Involvement of gicerin, a cell adhesion molecule, in development and regeneration of oviduct, and metastasis of oviductal adenocarcinomas of the chicken. Exp Cell Res 247: 329-338, 1999.

13. Tsukamoto Y, Taira E, Nakane Y, Tsudzuki M, Kohama K, Amin H, Miki N and Sasaki F: Expression of gicerin, a cell adhesion molecule, in the abnormal retina in silver plumage color mutation of Japanese quail (Coturnix japonica). Neurosci Lett 266: 53-56, 1999.

14. Choung CM, Randall BW, Sheree TB and Ting-Xin J: Early events during avian skin appendage regeneration: dependence on epithelial-mesenchymal interaction and order of molecular reappearance. J Invest Dermatol 107: 639-646, 1996.

15. Gallin WJ and Hepperle B: Burn healing in organ cultures of embryonic chicken skin: a model system. Burns 24: 613-620, 1998.

16. Hiroi S, Tsukamoto Y, Sasaki F, Miki N and Taira E: Involvement of gicerin, a cell adhesion molecule, in development and regeneration of chick sciatic nerve. FEBS Lett 554: 311-314, 2003.

17. Tsukamoto Y, Taira E, Miki N and Sasaki F: The role of gicerin, a novel cell adhesion molecule, in development, regeneration and neoplasia. Histol Histopathol 16: 563-571, 2001.

18. Nanchahal $\mathrm{J}$ and Ward CM: New grafts for old? A review of alternatives to autologous skin. Br J Plast Surg 45: 354-363, 1992.

19. Tsuchiya S, Tsukamoto Y, Furuya M, Hiroi S, Miki N, Sasaki F and Taira E: Gicerin, a cell adhesion molecule, promotes the metastasis of lymphoma cells of the chicken. Cell Tissue Res 314: 389-397, 2003. 